\title{
Mediators of racial disparities in mortality rates after traumatic brain injury in childhood: data from the Trauma Quality Improvement Program
}

\author{
Joseph Piatt, MD, MAS \\ Division of Neurosurgery, Nemours/Alfred I. duPont Hospital for Children, Wilmington, Delaware; and Departments of \\ Neurological Surgery and Pediatrics, Sidney Kimmel Medical College, Thomas Jefferson University, Philadelphia, Pennsylvania
}

\begin{abstract}
OBJECTIVE Social disparities in healthcare outcomes are almost ubiquitous, and trauma care is no exception. Because social factors cannot cause a trauma outcome directly, there must exist mediating causal factors related to the nature and severity of the injury, the robustness of the victim, access to care, or processes of care. Identification of these causal factors is the first step in the movement toward health equity.
\end{abstract}

METHODS A noninferiority analysis was undertaken to compare mortality rates between Black children and White children after traumatic brain injury (TBI). Data were derived from the Trauma Quality Improvement Program (TQIP) registries for the years 2014 through 2017. Inclusion criteria were age younger than 19 years and head Abbreviated Injury Scale scores of 4,5 , or 6 . A noninferiority margin of $10 \%$ was preselected. A logistic regression propensity score model was developed to distinguish Black and White children based on all available covariates associated with race at $p<0.10$. Stabilized inverse probability weighting and a one-tailed $95 \% \mathrm{Cl}$ were used to test the noninferiority hypothesis.

RESULTS There were 7273 observations of White children and 2320 observations of Black children. The raw mortality rates were $15.6 \%$ and $22.8 \%$ for White and Black children, respectively. The final propensity score model included 31 covariates. It had good fit (Hosmer-Lemeshow $\chi^{2}=7.1604, \mathrm{df}=8 ; \mathrm{p}=0.5194$ ) and good discrimination (c-statistic = 0.752). The adjusted mortality rates were $17.82 \%$ and $17.79 \%$ for White and Black children, respectively. The relative risk was 0.9986 , with a confidence interval upper limit of 1.0865 . The relative risk corresponding to the noninferiority margin was 1.1. The hypothesis of noninferiority was supported.

CONCLUSIONS Data captured in the TQIP registries are sufficient to explain the observed racial disparities in mortality after TBI in childhood. Speculations about genetic or epigenetic factors are not supported by this analysis. Discriminatory care may still be a factor in TBI mortality disparities, but it is not occult. If it exists, evidence for it can be sought among the data included in the TQIP registries.

https://thejns.org/doi/abs/10.3171/2020.5.PEDS20336

KEYWORDS disparities; outcome; race; trauma; traumatic brain injury; mortality

$\mathrm{S}$ OCIAL disparities in healthcare outcomes based on race and economic status are discovered almost wherever they are sought. For many conditions, the explanations for outcome disparities are readily understood in terms of environmental quality or access to care. For some conditions, discriminatory practices by providers may play a role. Identifying and-so far as possibleaddressing the mediators of disparities in healthcare outcomes are moral imperatives for contemporary physicians.

Of particular concern for surgeons, disparities in trauma mortality have been demonstrated consistently for both adults and children. The mediators of trauma outcome disparities ought to be apparent or measurable. Particularly in childhood, patients have few comorbidities prior to injury. The mechanism of the injury is usually well defined. There are validated metrics for the severity of injury. Time from injury to definitive care can be measured. There are no insurance barriers to the acute care of children with life-threatening injuries. In organized trauma systems, there are benchmarks for processes of care that are routinely recorded, and such systems generally cultivate a culture of caring and accountability that makes less room for

ABBREVIATIONS ACS = American College of Surgeons; AIS = Abbreviated Injury Scale; GCS = Glasgow Coma Scale; ICP = intracranial pressure; PUF = Participant User Files; RCT = randomized controlled trial; TBI = traumatic brain injury; TQIP = Trauma Quality Improvement Program; TQP = Trauma Quality Programs.

SUBMITTED April 27, 2020. ACCEPTED May 11, 2020.

INCLUDE WHEN CITING Published online July 31, 2020; DOI: 10.3171/2020.5.PEDS20336. 
discriminatory behavior by providers than occurs in some other settings.

For many years, trauma systems verified by the Committee on Trauma of the American College of Surgeons (ACS) have submitted defined data elements prospectively to the National Trauma Data Bank for research purposes. Since 2014 the Trauma Quality Improvement Program (TQIP) of the ACS has expanded these reporting requirements to include additional data describing access to care and processes of care for purposes of quality improvement. The TQIP data set is therefore an ideal environment in which to pose the question of whether social disparities in trauma mortality can be explained on the basis of recognized outcome determinants.

Studies of the social determinants of trauma outcomes have often followed a set pattern: a multivariate statistical model is constructed using predictors that have a demonstrated univariate association with the outcome or are known on the basis of expert knowledge to be important determinants. To this model are added social factors such as race, ethnicity, or measures of economic status. Either these social factors improve the model, in which case a social disparity is identified, or they do not. The study reported here has taken a different approach modeled after contemporary comparative effectiveness research with observational data. ${ }^{1-3}$ It views White race and Black race as "treatments" and employs propensity scoring to test the hypothesis of a causal treatment effect.

\section{Methods \\ Study Design}

A retrospective cohort study of mortality after traumatic brain injury (TBI) was undertaken using data from the TQIP for the years 2014 through 2017 (ACS Committee on Trauma. Trauma Quality Programs [TQP] Participant User Files [PUF]. Version 1.0. American College of Surgeons; 2019). Mortality rates for Black and White children were compared because these two social categories consistently exhibit large outcome differences. Black and White races were taken as "treatments" for a propensity score analysis. A binary logistic regression model was developed to distinguish Black children from White children. Every covariate from the TQIP data set having a univariate association with race, at the $\mathrm{p}<0.10$ level, was entered into the model. Stabilized inverse probability weighting was then employed to compare mortalities after adjustment for all the covariates that distinguished Black race from White race. ${ }^{3}$

That adjusted survival for Black children after TBI is worse than it is for White children was taken as the null hypothesis, and a noninferiority analysis was planned. A $10 \%$ greater mortality rate for Black children was predetermined as the noninferiority margin. A one-tailed hypothesis test with an $\alpha=0.05$ was planned. That is, if the upper limit of the one-tailed $95 \%$ CI for the adjusted relative risk of death among Black children were to fall below the noninferiority margin of 1.1, the null hypothesis would be rejected, and the conclusion that adjusted survival among Black children is not worse than that among White children would be accepted. Confidence intervals for relative risk were calculated using the PROC FREQ procedure in SAS and checked by Poisson bootstrap with 10,000 resamplings.

\section{Inclusion and Exclusion Criteria}

TBI was defined by a head Abbreviated Injury Scale (AIS) score of 4, 5, or 6 . Ages ranged up to 18 years, inclusive. Observations coded for discharge from the emergency department and for transfer to another short-term acute care facility were excluded.

\section{Data Organization}

The TQIP includes two fields for race and a field for ethnicity. Race was categorized in a hierarchical fashion as "Hispanic," "White," "Black," or "other." If ethnicity was coded as Hispanic, the case was so categorized. If the only racial coding was White, the case was so categorized. If either race field was coded as Black, the case was so categorized. All other codings of race and ethnicity were categorized as other.

Age is designated 1-18 years in the TQIP. There were no observations with an age of 0 , and for a substantial number of observations age was missing. Weight was not nearly so sparse as age, so ages were reassigned for missing age and for age of 1 year: observations with weight $\leq$ $9 \mathrm{~kg}$ were assigned an age of 0 years, and an age of 1 year was assigned for a weight of $\leq 16 \mathrm{~kg}$ (the 99th percentile for observations originally coded for an age of 1 year). Age was then categorized as "infant" (0 years), "toddler" (1-3 years), "child" (4 through 13 years), and "teen" (14 through 18 years).

Cases coded as "Medicaid," "Medicare," "self-pay," or "not billed" were categorized as "public" insurance. Cases coded as "other government" were included in the "commercial" category, as they were likely to be military dependents or beneficiaries of other government employees.

Covariates were reorganized to harmonize the annual TQIP data sets and to minimize numbers of categories while highlighting associations with mortality. All quantitative covariates were reduced to categories, as for age so also for Injury Severity Score, systolic blood pressure in the emergency department, arrival in the emergency department, number of protective devices, blood product administration, number of diagnoses, and number of procedures. Missing data were designated as a separate category or folded into a category with a similar mortality rate. Selected qualitative clinical factors were defined by searching diagnostic and procedural codes.

The ACS stipulates the following acknowledgment: "The content reproduced from the TQP PUF remains the full and exclusive copyrighted property of the American College of Surgeons. The American College of Surgeons is not responsible for any claims arising from works based on the original data, text, tables, or figures." No content from the TQP PUF has been reproduced in this manuscript.

Data analysis was performed using SAS version 9.4 (SAS Institute, Inc.). This project was judged not to be research of human subjects by the Office of Human Research of Thomas Jefferson University. 


\section{Results}

There were 9772 cases meeting the study definition of TBI, 6520 males (66.7\%) and 3252 females (33.3\%). Survival data were unavailable for 179 cases. Among cases with survival data there were 7273 White children and 2320 Black children. There were 1661 deaths (17.3\%). Intracranial monitors were coded in $18.6 \%$ of cases. Other neurosurgical procedures were recorded for $13.9 \%$ of the cases within the first 4 days of admission and in $2.8 \%$ of cases at later times in the hospital course.

Covariates entered into the propensity score model are presented in the Table 1, with adjusted odds ratios and confidence intervals describing their associations with Black race as compared to White race. Black race was associated with infancy and with missing age data. White race was associated with the teenage group. Black race was associated with metrics of more severe injury, as reflected in Glasgow Coma Scale (GCS) scores, AIS scores, number of diagnoses, number of procedures, and number of ventilator days, but not by Injury Severity Scores. Black race was associated with violent mechanisms of injury, including suicide. On the other hand, in this multivariate model, White race was associated with abuse. (In univariate analysis, the prevalence of abuse was slightly higher among Black children than White children, $11.2 \%$ as compared to 9.2\% [data not shown].) White race was associated with coding for protective devices, airbags, and ICP monitors. Black children were relatively underrepresented among observations with positive alcohol screening and among observations with missing data for this screening. They had higher rates of public insurance. They were less often brought to the hospital by private vehicle and less often transferred from another hospital. They were more commonly treated in the largest hospitals and in university hospitals than White children. Black children were overrepresented among observations from hospitals unverified as trauma centers by the ACS and among observations with missing data for ACS verification.

The goal of propensity score weighting is to balance between the treatments the impacts of important confounders. The balancing of selected important prognostic factors is presented in Table 2.

The raw mortality rates were $15.6 \%$ and $22.8 \%$ for White and Black children, respectively. Allowing for missing mortality data, the number of raw observations was 9593 . Stabilized inverse probability weighting yielded 9591.3 effective observations. The adjusted mortality rates were $17.82 \%$ and $17.79 \%$ for White and Black children, respectively. The relative risk was 0.9986 with a one-tailed 95\% CI upper limit of 1.09. The bootstrap limit was 1.10. The relative risk corresponding to the noninferiority margin was 1.10 by definition. The hypothesis of noninferiority was supported.

\section{Discussion}

Associations of social factors with trauma outcomes have been studied extensively in the United States for more than a quarter century. ${ }^{4}$ Mechanisms and severities of injury, age distributions, comorbidities, access to care, and processes of care have been recognized to differ
TABLE 1. Associations with Black race in the multivariate propensity score model

\begin{tabular}{|c|c|c|}
\hline Covariate/Category & Adjusted OR (95\% Cl) & $\mathrm{p}$ Value \\
\hline Age group & & $<0.0001$ \\
\hline Infant & $1.23(1.04-1.46)$ & \\
\hline Toddler & $(0.83-1.21)$ & \\
\hline Child & Reference & \\
\hline Teen & $0.67(0.57-0.78)$ & \\
\hline Missing & $1.35(1.09-1.68)$ & \\
\hline GCS motor score & & $<0.0001$ \\
\hline 6 & Reference & \\
\hline 5 & $1.41(1.14-1.73)$ & \\
\hline 4 & $1.48(1.17-1.88)$ & \\
\hline 3 & $1.15(0.80-1.66)$ & \\
\hline 2 & $1.45(0.97-2.17)$ & \\
\hline 1 & $1.45(1.22-1.73)$ & \\
\hline Missing & $0.77(0.59-1.01)$ & \\
\hline Violence & & $<0.0001$ \\
\hline No & Reference & \\
\hline Yes & $2.36(1.62-3.45)$ & \\
\hline Abuse & & $<0.0001$ \\
\hline No & Reference & \\
\hline Yes & $0.38(0.25-0.57)$ & \\
\hline Protective devices & & $<0.0001$ \\
\hline None & Reference & \\
\hline Some & $0.64(0.54-0.77)$ & \\
\hline Airbag & & $<0.0001$ \\
\hline No & $1.49(1.04-2.13)$ & \\
\hline Yes & Reference & \\
\hline Missing & $0.45(0.34-0.58)$ & \\
\hline Payer & & $<0.0001$ \\
\hline Public & Reference & \\
\hline Commercial & $0.35(0.31-0.39)$ & \\
\hline Missing & $0.80(0.55-1.15)$ & \\
\hline ICP monitor & & 0.0035 \\
\hline No & Reference & \\
\hline Yes & $0.78(0.67-0.92)$ & \\
\hline Missing & $1.28(0.93-1.76)$ & \\
\hline Self-transport & & 0.0043 \\
\hline No & Reference & \\
\hline Yes & $0.76(0.63-0.92)$ & \\
\hline Diagnoses & & 0.0053 \\
\hline 1st quartile & Reference & \\
\hline 2nd or 3rd quartile & $0.90(0.79-1.03)$ & \\
\hline 4th quartile & $0.73(0.61-0.89)$ & \\
\hline Ventilator days & & 0.0064 \\
\hline None/missing & Reference & \\
\hline $1-4$ & $1.24(1.05-1.46)$ & \\
\hline$>5$ & $1.40(1.13-1.73)$ & \\
\hline
\end{tabular}

CONTINUED ON PAGE $479 »$ 
» CONTINUED FROM PAGE 478

TABLE 1. Associations with Black race in the multivariate propensity score model

\begin{tabular}{|c|c|c|}
\hline Covariate/Category & Adjusted OR $(95 \% \mathrm{Cl})$ & $p$ Value \\
\hline Alcohol & & 0.0124 \\
\hline Negative & Reference & \\
\hline Positive & $0.58(0.39-0.84)$ & \\
\hline Missing & $0.45(0.34-0.58)$ & \\
\hline Procedures & & 0.0387 \\
\hline None & Reference & \\
\hline $1-4$ & $1.20(0.91-1.57)$ & \\
\hline $5-14$ & $1.36(1.03-1.80)$ & \\
\hline$>14$ & $1.50(1.10-2.05)$ & \\
\hline Head AIS score & & 0.0470 \\
\hline 4 & Reference & \\
\hline 5 & $0.86(0.74-0.99)$ & \\
\hline 6 & $1.31(0.70-2.45)$ & \\
\hline Systolic pressure & & 0.0787 \\
\hline Low & $1.25(0.98-1.58)$ & \\
\hline Midrange & Reference & \\
\hline High & $1.25(0.98-1.59)$ & \\
\hline Missing & $0.93(0.74-1.17)$ & \\
\hline ED disposition & & 0.2027 \\
\hline Dead & $0.84(0.57-1.23)$ & \\
\hline Floor & Reference & \\
\hline OR or ICU & $0.88(0.75-1.04)$ & \\
\hline Missing & $0.75(0.57-0.99)$ & \\
\hline Sex & & 0.2174 \\
\hline Female & $0.93(0.54-1.04)$ & \\
\hline Male & Reference & \\
\hline ISS & & 0.2873 \\
\hline Missing or 1-17 & Reference & \\
\hline $18-29$ & $1.09(0.93-1.27)$ & \\
\hline$>29$ & $1.20(0.96-1.50)$ & \\
\hline Drug screen & & 0.3112 \\
\hline Missing or negative & Reference & \\
\hline Positive & $0.90(0.73-1.10)$ & \\
\hline Open injury & & 0.3232 \\
\hline Yes & $1.09(0.92-1.30)$ & \\
\hline No & Reference & \\
\hline Penetrating injury & & 0.6892 \\
\hline Yes & $1.09(0.71-1.69)$ & \\
\hline No & Reference & \\
\hline Blood products & & 0.7767 \\
\hline None & Reference & \\
\hline$<2 \mathrm{~L}$ & $0.95(0.71-1.28)$ & \\
\hline$>2 \mathrm{~L}$ & $1.01(0.74-1.38)$ & \\
\hline Inbound transfer & & $<0.0001$ \\
\hline No & Reference & \\
\hline Yes & $0.73(0.64-0.82)$ & \\
\hline
\end{tabular}

CONTINUED IN NEXT COLUMN »
» CONTINUED FROM PREVIOUS COLUMN

TABLE 1. Associations with Black race in the multivariate propensity score model

\begin{tabular}{|c|c|c|}
\hline Covariate/Category & Adjusted OR $(95 \% \mathrm{Cl})$ & $p$ Value \\
\hline Hospital bed size & & $<0.0001$ \\
\hline$\leq 200$ & $0.79(0.62-1.01)$ & \\
\hline $201-400$ & $0.86(0.73-1.02)$ & \\
\hline $401-600$ & $0.63(0.54-0.73)$ & \\
\hline$>600$ & Reference & \\
\hline Teaching status & & $<0.0001$ \\
\hline University & Reference & \\
\hline Community & $0.65(0.56-0.75)$ & \\
\hline Nonteaching & $0.62(0.46-0.84)$ & \\
\hline Missing & $0.55(0.28-1.09)$ & \\
\hline ACS level & & $<0.0001$ \\
\hline I & Reference & \\
\hline II & $0.69(0.37-1.28)$ & \\
\hline Not applicable & $1.51(1.23-1.85)$ & \\
\hline Missing & $1.50(1.24-1.80)$ & \\
\hline State level & & 0.0111 \\
\hline I & Reference & \\
\hline II & $1.19(0.76-1.88)$ & \\
\hline Not applicable & $0.83(0.69-1.00)$ & \\
\hline Missing & $0.78(0.64-0.94)$ & \\
\hline ACS pediatric level & & 0.3165 \\
\hline 1 & Reference & \\
\hline II & $0.93(0.72-1.21)$ & \\
\hline Not applicable & $1.08(0.92-1.26)$ & \\
\hline Missing & $0.88(0.74-1.05)$ & \\
\hline State pediatric level & & 0.4629 \\
\hline 1 & Reference & \\
\hline$\|$ & $0.95(0.70-1.29)$ & \\
\hline Not applicable & $1.11(0.94-1.30)$ & \\
\hline Missing & $0.96(0.81-1.13)$ & \\
\hline
\end{tabular}

ED = emergency department; ISS = Injury Severity Score; OR = operating room.

among socially defined groups of patients..$^{5-8}$ Of particular concern has been the treatment of less socially privileged groups at worse-performing hospitals. ${ }^{9-11}$ Most attempts at multivariate analysis have found persisting, unexplained associations of race, ethnicity, and economic status with mortality and other outcomes, such as transfer to rehabilitation and functional recovery. ${ }^{6,12-20}$ Not all studies concur, $, 21,22,23$ and social status may not have the same association with outcome in health systems with uniform insurance coverage. ${ }^{24-26}$ In some investigations, controlling for economic status has diminished or eliminated associations of outcomes with race and ethnicity. ${ }^{27-30}$

The prospective randomized controlled trial (RCT) is the accepted method for establishing causality, but even an $\mathrm{RCT}$ rests on an assumption that randomization balances the distribution of measured and unmeasured confounders between the control and the experimental arms. In recent 
decades, statistical methods have appeared that attempt to extract evidence for causality from observational data. Propensity scoring is a statistical model estimating the probability that an individual subject receives one treatment or another based on the associations of the treatments with other measured covariates. The propensity score can be used to match subjects receiving different treatments, or, as in the current study, it can be used to calculate weights that balance the effects of the measured covariates between the treatments. Like the RCT, the propensity score method rests on the assumption of the absence of unmeasured confounders. If the current propensity score analysis had demonstrated a causal relationship between race and mortality, the question of unmeasured confounders would be salient. As it established the noninferiority of the survival of Black children, considerable imagination is required to posit the existence of important unmeasured confounders that precisely equalize the mortality rates of the two treatment groups.

In the current study, race was taken to be a treatment, and Black race by itself, apart from all of the other factors with which it is associated in the TQIP, was determined not to be a cause of greater mortality after childhood TBI. As the higher mortality rate of Black children is an undisputed fact, the study conclusion means that the explanation for the excessive mortality lies somewhere among the covariates included in the propensity score model. Marked racial differences were evident in numerous important prognostic factors, including age group, GCS motor score, and violent mechanisms of injury. Greater numbers of procedures and ventilator days indicated that surviving Black children had more severe and complicated injuries. Vehicular injuries of Black children were less often coded for airbags. Lower rates of protective device use among Black children are more difficult to interpret, as the possibility of protective device use is a function of the mechanism of injury. As has been noted in the past, there were racial differences in hospital destinations and how children arrived at them. In the 2017 edition of the TQIP, the ACS dropped the facility code that had been present in previous years, so no analysis of facility performance was undertaken in the current study. Nevertheless, the overrepresentation of Black children at hospitals in the "not applicable" and "data missing" categories with respect to ACS trauma verification reinforces this concern.

Conversely, factors not included in the model are not supported as causes of TBI outcome disparities: topics of speculation have included nutritional factors, ${ }^{31,32}$ varying levels of past exposure to toxic psychosocial stress, ${ }^{33,34}$ varying prevalences of gene polymorphisms critical for a response to injury, ${ }^{35-37}$ and epigenetic factors accumulated over generations of social adversity. ${ }^{38,39}$ Such factors seem to play no quantitatively important role in survival after childhood TBI.

Discriminatory practices have been recognized in trauma care, and they can be mitigated by adherence to guidelines ${ }^{40}$ The current study does not eliminate a role for discriminatory care, but it focuses efforts to eliminate discriminatory care on processes recorded-or not recorded-in the TQIP registry. For example, as noted in the discussion of the propensity score model, observations
TABLE 2. Balance produced by propensity score stabilized inverse probability weighting between Black and White races for selected important covariates associated with death

\begin{tabular}{|c|c|c|c|}
\hline Covariate/Category & Black & White & $p$ Value \\
\hline Age group & & & 0.7340 \\
\hline Infant & $474.2(20.2 \%)$ & $1409.7(19.0 \%)$ & \\
\hline Toddler & $210.0(8.9 \%)$ & $666.9(9.0 \%)$ & \\
\hline Child & $715.9(30.5 \%)$ & $2319.3(31.3 \%)$ & \\
\hline Teen & $801.5(34.1 \%)$ & $2571.8(34.7 \%)$ & \\
\hline Missing & $149.1(6.3 \%)$ & $451.2(6.1 \%)$ & \\
\hline GCS motor score & & & 0.9230 \\
\hline 6 & $1193.5(58.0 \%)$ & $3736.8(58.2 \%)$ & \\
\hline 5 & $178.7(8.7 \%)$ & $579.4(9.0 \%)$ & \\
\hline 4 & $139.6(6.8 \%)$ & $404.9(6.3 \%)$ & \\
\hline 3 & $56.6(2.8 \%)$ & $159.5(2.5 \%)$ & \\
\hline 2 & $35.1(1.7 \%)$ & $118.9(1.9 \%)$ & \\
\hline 1 & $1426.3(22.2 \%)$ & $453.8(22.1 \%)$ & \\
\hline Head AIS score & & & 0.9317 \\
\hline 4 & $1398.7(59.5 \%)$ & $4445.1(59.9 \%)$ & \\
\hline 5 & $937.6(39.9 \%)$ & $2930.3(39.5 \%)$ & \\
\hline 6 & $14.4(0.6 \%)$ & $43.5(0.6 \%)$ & \\
\hline ISS & & & 0.7045 \\
\hline Missing or 1-17 & $734.3(31.2 \%)$ & $2384.9(32.2 \%)$ & \\
\hline $18-29$ & $1065.0(45.3 \%)$ & $3306.8(44.6 \%)$ & \\
\hline$>29$ & $551.4(23.5 \%)$ & $1727.3(23.3 \%)$ & \\
\hline Penetrating injury & & & 0.7377 \\
\hline No & $110.3(4.7 \%)$ & $360.8(4.9 \%)$ & \\
\hline Yes & $2240.4(95.3 \%)$ & $7058.1(95.1 \%)$ & \\
\hline Violence & & & 0.2638 \\
\hline No & $1948.4(82.9 \%)$ & $6221.9(83.9 \%)$ & \\
\hline Yes & $402.3(17.1 \%)$ & $1197.0(16.1 \%)$ & \\
\hline Abuse & & & 0.1821 \\
\hline No & $2096.6(89.2 \%)$ & $6687.6(90.1 \%)$ & \\
\hline Yes & $254.1(10.8 \%)$ & $731.4(9.9 \%)$ & \\
\hline ICP monitoring & & & 0.4023 \\
\hline No & $1711.4(72.8 \%)$ & $5391.2(72.7 \%)$ & \\
\hline Yes & $452.5(19.3 \%)$ & $1377.7(18.6 \%)$ & \\
\hline Missing & $186.8(8.0 \%)$ & $650.0(8.8 \%)$ & \\
\hline
\end{tabular}

with missing data were often different from the rest of the sample. Observations with missing data for race had a higher mortality rate than the rest of the sample, $21.3 \%$ compared to $16.6 \%$ (data not shown), and were suspiciously close to the raw mortality rate for Black children, $22.8 \%$. These data are not missing at random. That no race designation was recorded for these cases cannot have contributed directly to clinical outcomes, but this discrepancy may reflect other discriminatory processes in trauma system operations with more serious consequences. Another example of more direct concern in the multivariate propensity score model is the apparent underutilization of intracranial pressure (ICP) monitors among Black children. In the univariate analysis, ICP monitoring was actually 
more prevalent among Black children (data not shown). The resolution of this paradox may be that, even though ICP monitoring was more common among Black children, it was not as common as expected in the context of the other factors distinguishing Black and White children with TBI. Interpretation of such observations is clearly very challenging. Intentional prospective investigation may be necessary to elucidate questions of discriminatory care, but intentional investigation is likely to have the happy effect of suppressing it. That the question of discriminatory care might be settled by its disappearance is a worthy goal for future research.

\section{Limitations}

The TQIP data were collected prospectively at the time of hospital discharge by trained registrars in trauma programs verified by the ACS for the purposes of quality improvement. For this reason, the TQIP promised to be an ideal data source for studies of the mediators of social disparities in trauma-related mortality, but for the same reason, conclusions drawn from TQIP may not be generalizable to trauma care in other settings in the United States.

\section{Conclusions}

In this study of data from the TQIP, Black children suffered greater mortality after TBI than White children, but that disparity was shown to be mediated by covariates captured in the TQIP data set, not by some intrinsic feature of race and not by factors outside the scope of the TQIP. Efforts to mitigate social trauma outcome disparities can be focused most profitably on the covariates recorded in TQIP.

\section{Acknowledgments}

I thank Zachary Gandee for administrative assistance.

\section{References}

1. Curtis LH, Hammill BG, Eisenstein EL, et al. Using inverse probability-weighted estimators in comparative effectiveness analyses with observational databases. Med Care. 2007; 45(10)(suppl 2):S103-S107.

2. Rosenbaum PR, Rubin DB. The central role of the propensity score in observational studies for causal effects. Biometrika. 1983;70(1):41-55.

3. Xu S, Ross C, Raebel MA, et al. Use of stabilized inverse propensity scores as weights to directly estimate relative risk and its confidence intervals. Value Health. 2010;13(2): 273-277.

4. Goins WA, Rodriguez A, Dunham CM, Shankar BS. Blackwhite disparities in blunt trauma. J Natl Med Assoc. 1993; 85(8):601-607.

5. Marcin JP, Schembri MS, He J, Romano PS. A populationbased analysis of socioeconomic status and insurance status and their relationship with pediatric trauma hospitalization and mortality rates. Am J Public Health. 2003;93(3):461-466.

6. Rangel SJ, Martin CA, Brown RL, et al. Alarming trends in the improper use of motor vehicle restraints in children: implications for public policy and the development of racebased strategies for improving compliance. J Pediatr Surg. 2008;43(1):200-207.

7. Shafi S, de la Plata CM, Diaz-Arrastia R, et al. Ethnic disparities exist in trauma care. J Trauma. 2007;63(5):1138-1142.
8. Wood JN, Hall M, Schilling S, et al. Disparities in the evaluation and diagnosis of abuse among infants with traumatic brain injury. Pediatrics. 2010;126(3):408-414.

9. Glance LG, Osler TM, Mukamel DB, et al. Trends in racial disparities for injured patients admitted to trauma centers. Health Serv Res. 2013;48(5):1684-1703.

10. Haider AH, Hashmi ZG, Zafar SN, et al. Minority trauma patients tend to cluster at trauma centers with worse-thanexpected mortality: can this phenomenon help explain racial disparities in trauma outcomes? Ann Surg. 2013;258(4): 572-581.

11. Hicks CW, Hashmi ZG, Hui X, et al. Explaining the paradoxical age-based racial disparities in survival after trauma: the role of the treating facility. Ann Surg. 2015;262(1):179-183.

12. Falcone RA Jr, Brown RL, Garcia VF. Disparities in child abuse mortality are not explained by injury severity. J Pediatr Surg. 2007;42(6):1031-1037.

13. Falcone RA Jr, Brown RL, Garcia VF. The epidemiology of infant injuries and alarming health disparities. J Pediatr Surg. 2007;42(1):172-177.

14. Falcone RA Jr, Martin C, Brown RL, Garcia VF. Despite overall low pediatric head injury mortality, disparities exist between races. J Pediatr Surg. 2008;43(10):1858-1864.

15. Haider AH, Efron DT, Haut ER, et al. Black children experience worse clinical and functional outcomes after traumatic brain injury: an analysis of the National Pediatric Trauma Registry. J Trauma. 2007;62(5):1259-1263.

16. Hakmeh W, Barker J, Szpunar SM, et al. Effect of race and insurance on outcome of pediatric trauma. Acad Emerg Med. 2010;17(8):809-812.

17. Lee FA, Hervey AM, Sattarin A, et al. The impact of payer source on trauma outcomes in a pediatric population. Hosp Pediatr. 2017;7(3):171-176.

18. Martin CA, Care M, Rangel EL, et al. Severity of head computed tomography scan findings fail to explain racial differences in mortality following child abuse. Am J Surg. 2010; 199(2):210-215.

19. Meagher AD, Beadles CA, Doorey J, Charles AG. Racial and ethnic disparities in discharge to rehabilitation following traumatic brain injury. J Neurosurg. 2015;122(3):595-601.

20. Nirula R, Nirula G, Gentilello LM. Inequity of rehabilitation services after traumatic injury. J Trauma. 2009;66(1): 255-259.

21. Howard I, Joseph JG, Natale JE. Pediatric traumatic brain injury: Do racial/ethnic disparities exist in brain injury severity, mortality, or medical disposition? Ethn Dis. 2005;15(4 Suppl 5):S5-51-S5-56.

22. Lee SL, Yaghoubian A, Stark R, et al. Are there racial disparities in the use of restraints and outcomes in children after motor vehicle crashes? J Pediatr Surg. 2012;47(6):1192-1195.

23. Osler T, Glance LG, Li W, et al. Re: Trauma care does not discriminate: the association of race and health insurance with mortality following traumatic injury. J Trauma Acute Care Surg. 2015;79(3):516-517.

24. Chaudhary MA, Sharma M, Scully RE, et al. Universal insurance and an equal access healthcare system eliminate disparities for black patients after traumatic injury. Surgery. 2018;163(4):651-656.

25. Leow JJ, Lim VW, Lingam P, et al. Ethnic disparities in trauma mortality outcomes. World J Surg. 2014;38(7):1694-1698.

26. Moore L, Turgeon AF, Sirois MJ, et al. Influence of socioeconomic status on trauma center performance evaluations in a Canadian trauma system. J Am Coll Surg. 2011;213(3): 402-409.

27. Gerry JM, Weiser TG, Spain DA, Staudenmayer KL. Uninsured status may be more predictive of outcomes among the severely injured than minority race. Injury. 2016;47(1): 197-202.

28. Haider AH, Chang DC, Efron DT, et al. Race and insurance 
status as risk factors for trauma mortality. Arch Surg. 2008; 143(10):945-949.

29. Rosen H, Saleh F, Lipsitz SR, et al. Lack of insurance negatively affects trauma mortality in US children. $J$ Pediatr Surg. 2009;44(10):1952-1957.

30. Salim A, Ottochian M, DuBose J, et al. Does insurance status matter at a public, level I trauma center? J Trauma. 2010; 68(1):211-216.

31. Bechard LJ, Duggan C, Touger-Decker R, et al. Nutritional status based on body mass index is associated with morbidity and mortality in mechanically ventilated critically ill children in the PICU. Crit Care Med. 2016;44(8):1530-1537.

32. McNally JD, Nama N, O'Hearn K, et al. Vitamin D deficiency in critically ill children: a systematic review and metaanalysis. Crit Care. 2017;21(1):287.

33. Lê-Scherban F, Wang X, Boyle-Steed KH, Pachter LM. Intergenerational associations of parent adverse childhood experiences and child health outcomes. Pediatrics. 2018;141(6): e20174274.

34. Oh DL, Jerman P, Silvério Marques S, et al. Systematic review of pediatric health outcomes associated with childhood adversity. BMC Pediatr. 2018;18(1):83.

35. Jha RM, Koleck TA, Puccio AM, et al. Regionally clustered $A B C C 8$ polymorphisms in a prospective cohort predict cerebral oedema and outcome in severe traumatic brain injury. $J$ Neurol Neurosurg Psychiatry. 2018;89(11):1152-1162.

36. Osier ND, Conley YP, Okonkwo DO, Puccio AM. Variation in candidate traumatic brain injury biomarker genes are associated with gross neurological outcomes after severe traumatic brain injury. J Neurotrauma. 2018;35(22):2684-2690.
37. Yue JK, Winkler EA, Rick JW, et al. DRD2 C957T polymorphism is associated with improved 6-month verbal learning following traumatic brain injury. Neurogenetics. 2017;18(1): 29-38.

38. Costa DL, Yetter N, DeSomer H. Intergenerational transmission of paternal trauma among US Civil War ex-POWs. Proc Natl Acad Sci U S A. 2018;115(44):11215-11220.

39. Youssef NA, Lockwood L, Su S, et al. The effects of trauma, with or without PTSD, on the transgenerational DNA methylation alterations in human offsprings. Brain Sci. 2018;8(5): E83.

40. Rangel EL, Cook BS, Bennett BL, et al. Eliminating disparity in evaluation for abuse in infants with head injury: use of a screening guideline. J Pediatr Surg. 2009;44(6):1229-1235.

\section{Disclosures}

The author reports no conflict of interest concerning the materials or methods used in this study or the findings specified in this paper.

\section{Correspondence}

Joseph Piatt: Nemours/Alfred I. duPont Hospital for Children, Wilmington, DE.jpiatt@nemours.org. 